\title{
LA CARRERA DOCENTE EN LA UNIVERSIDAD NACIONAL DE ROSARIO
}

\author{
Rosana Schanzer (UNR)* \\ Cecilia Muruaga (UNR)**
}

\section{Resumen}

La Universidad Nacional de Rosario (UNR) cuenta con un dispositivo de evaluación permanente para la docencia; a partir del año 1989 la Honorable Asamblea Universitaria definió, en el artículo 60 del Estatuto, la permanencia de los docentes en sus cargos concursados y dejó sin efecto el concurso periódico. En la actualidad se rige por la Ordenanza $N^{\circ} 602 / 00$, producto de sucesivas modificaciones operadas sobre la ordenanza 526/92. La carrera docente normalizó su funcionamiento a partir del año 2000 y resulta necesario evaluar su desenvolvimiento en el ámbito de gestión, a fin de considerar las sugerencias de los actores institucionales implicados. La presente investigación se genera a partir de la detección de núcleos problemáticos en su desarrollo: la organización del sistema de información; el marco normativo; los instrumentos que completa el docente evaluado, y pretende ser una contribución para la generación de conocimientos sobre evaluación de la profesión docente en la Educación Superior que constituyan un aporte para los equipos que participan en su gestión en los ejes: evaluación, planificación y docencia universitaria.

\section{Palabras clave}

Evaluación carrera docente - Gestión - Núcleos problemáticos - Actores institucionales - Educación superior.

\section{Abstract}

The Universidad Nacional de Rosario (UNR) has a permanent evaluation mechanism for teaching; from the year 1989, the Honorable University Assembly

Especialista en Gestión y Conducción del Sistema Educativo y sus instituciones. FLACSO (2009). Licenciada en Ciencia Política y Licenciada en RR.II. Facultad de Ciencia Política y RR.II. Universidad Nacional de Rosario.

" Diplomada Superior en Ciencias Sociales con mención en Ciencia Política. FLACSO. Costa Rica. Profesora de Enseñanza Media y Superior en Letras. Facultad de Humanidades y Artes. Universidad Nacional de Rosario. 
defined in the article 60 of the Statute, the permanence of teachers in their positions and resciended the regular competition. It is actually governed by the Ordinance $602 / 00$, the result of successive modifications operated on the ordinance $526 / 92$. The teaching career normalized its operation from year 2000 and it is necessary to evaluate its performance in the field of management, to consider the suggestions of institutional actors involved. This research was generated from the detection of problematic items in its development: the organization of information system, the policy framework, the evaluation instruments completed by the evaluated teachers, and pretends to be a contribution for the generation of knowledge about the evaluation of the teaching profesión in higher education and an input for teams envolved in managing of the teaching profesión in the areas: assessment, planning and university teaching.

\section{Key words:}

Evaluation of the teaching career - Management - Problematic items - Institutional actors - Higher education.

\section{Introducción}

La calidad de la Educación Superior depende en gran medida, aunque no únicamente, del nivel y la calificación de su personal docente, ya que asume la mayor parte de las tareas relevantes en la formación (Arrechavaleta Guarton, Trista Perez, 1992). Como aseguran estos autores, las relaciones que se establecen a partir de la consideración de la formación, distribución y utilización del personal académico, sirven de base para la elaboración del sistema de planes, la organización y la evaluación de la formación y la superación del personal docente. Esto pone en evidencia la importante relación que existe entre una planificación adecuada que contemple la distribución de los recursos humanos y su calificación y la evaluación docente. Desde esta perspectiva se espera que esta última tribute a un sistema de información que pueda al menos determinar:

- Los objetivos de formación (y superación) de acuerdo a las habilidades y conocimientos requeridos para cada cargo docente.

- Los requerimientos mínimos para el ingreso a la docencia.

- La determinación de las necesidades de formación para la superación individual del cuerpo docente y su articulación en políticas que organicen tales demandas e instrumenten programas para dar cuenta de ellas.

Desde otra perspectiva, se considera que la Educación Superior universitaria ha sufrido cambios en las últimas décadas del siglo pasado que varían la configuración actual de las universidades. Estos cambios suponen: mutilaciones del orden disciplinar al interdisciplinario; la mejora de la administración y consiguiente profesionalización de la gestión universitaria; la orientación de la 
matrícula hacia el mercado de trabajo como alternativa a elección vocacional del currículo; el crecimiento del sector privado y el retraimiento del sector público que no puede, sobre todo en países con desarrollo económico limitado, dar cuenta del crecimiento de la demanda del alumno.

Analizar el futuro de los sistemas educativos y los mecanismos destinados a mejorar su calidad y funcionamiento, muestran la necesidad de considerar los nuevos contextos que llevan a pensar la universidad pública en la actualidad.

El campo de la política universitaria argentina se puede pensar constituido por distintos actores que tienen distinto grado de fuerza a partir de la redefinición de las relaciones entre Ministerio y Universidades. Es un campo de fuerzas y tensiones que atraviesa modificaciones muy rápidas. Nos encontramos con grandes temas sin resolución, que están en proceso de renegociación: la gestión del presupuesto público, la gestión administrativa, la evaluación de la calidad, la capacidad habilitante de los títulos, el estímulo a la investigación científica, los mecanismos de evaluación.

La Universidad está sometida a presiones que vienen desde el mercado de trabajo, pero también existen presiones fuertes ejercidas desde el Estado que exige calificaciones importantes y fija criterios de evaluación de calidad que se alejan, en algunos casos, de los que necesariamente debe darse la institución.

El papel protagónico que universalmente pasa a tener el conocimiento en todos los procesos de la vida social en el presente influye en nuevas formas de producción, circulación y apropiación y evaluación del conocimiento en las instituciones de nivel superior. Es por ello que las mismas deben estar dispuestas a conocerse mejor y a evaluar ellas mismas el cumplimiento de sus responsabilidades, a través de evaluaciones que no estén basadas solamente en la eficiencia sino en la capacidad institucional para participar en la construcción de cambios de largo alcance, al mismo tiempo que participar en su construcción y saber adaptarse a los procesos cambiantes implicados en los nuevos conocimientos.

La Ley de Educación Superior prescribe un ordenamiento básico para establecer un sistema de evaluación de las universidades nacionales y establece algunos mecanismos básicos a partir de la constitución de la Comisión Nacional de Evaluación y Acreditación Universitaria.

En este marco, la "profesión académica" ha sufrido presiones producto de una combinación de factores económicos y demográficos. En los países más pobres el crecimiento de la matrícula ha estado acompañado por la disminución de los recursos, y los docentes asumen la necesidad de generarlos mediante financiamientos especiales para la investigación (Altbach, 2001).

Por otra parte, la definición de la tarea docente implica una distinción entre práctica pedagógica y práctica docente; la primera se refiere a la relación docente - alumnos - conocimiento, y se restringe a las relaciones áulicas, en 
tanto que la segunda incorpora elementos que configuran el campo laboral o profesional del profesor (Achilli, 2000). Se agrega, además, a esta discusión, la existencia de una tensión entre docencia e investigación; como indica la bibliografía sobre el tema, la investigación es un componente básico de la profesión docente en la medida en que produce conocimiento nuevo y asume un carácter transitivo hacia la formación del alumno. Así, el curriculo de las universidades se ha reorientado para lograr que la investigación no sea una "actividad casual" o de cierre al momento de la graduación. Se ha puesto en evidencia la necesidad de que el docente la incorpore como una actividad permanente en cada asignatura, para la resolución tanto de problemas teóricos como prácticos (Ruiz Luquez, 2000).

La formación docente del profesor universitario también es objeto de reflexiones, puesto que predomina un perfil de corte tradicional que privilegia la experticia disciplinar o directamente profesional, sin atender a la innovación de los procesos educativos; en este sentido la docencia sostiene un modelo reproductivo de los saberes, basado en una "praxis acrítica" (Hernandez Madrigal, 2003). El análisis de la práctica educativa se torna un componente fundamental para garantizar la calidad en el nivel superior lo que dependerá de las políticas institucionales para la consolidación de los cuerpos académicos. En este sentido la evaluación de las capacidades docentes debe estar al servicio de brindar, desde las instituciones, una oportunidad de mejora de su práctica profesional a partir de un proceso inclusivo, antes que de un sistema de control y fiscalización de sus tareas (Zabalza, 1990).

La evaluación institucional nos permite un interesante debate, en la medida que la aplicación práctica de sus resultados resulta de interés para las investigaciones sobre Educación Superior

Los diferentes enfoques acerca de la evaluación pueden entenderse en forma complementaria, en la medida que pretendan superar su visión como mero instrumento para la toma de decisiones. Esta mirada puede aplicarse a los distintos niveles y aspectos del proceso evaluativo y contribuye a responder a la demanda por la calidad educativa.

La evaluación requiere de una mirada amplia sobre la realidad que se pretende evaluar y su lectura final debe incluir tanto las consecuencias conforme a los objetivos planteados como aquellas que, no habiendo sido previstas, tengan incidencia en la institución o programa objeto de evaluación.

Vain (1998) sostiene que el desempeño de los docentes universitarios puede ser entendido como una práctica educativa que supone múltiples articulaciones y que al mismo tiempo se presenta como realidad compleja, dificultando en muchos casos su evaluación, ya que resulta necesario sistematizar la multiplicidad de información que pueda recabarse sobre esta realidad y organizarla de modo de viabilizar su interpretación. 
La implementación de la carrera docente supone la evaluación de cada docente, entendiéndose a la docencia como una actividad cotidiana desarrollada por actores, en tiempos y espacios específicos. En este sentido, es necesario abordar su evaluación desde sistemas lo suficientemente permeables como para contener su complejidad, teniendo en cuenta, según expresan Fernández Lamarra y Marquina (2008), que la profesión académica como objeto de investigación ha cobrado importancia en las últimas décadas, paralelamente al crecimiento y afianzamiento de la Educación Superior como objeto de investigación.

En las Consideraciones Generales de la Autoevaluación Institucional de la Universidad Nacional de Rosario (UNR) del año 2002 se plantea que "difícilmente pueda cuestionarse en la actualidad la necesidad de una transformación a fondo en la Educación Superior", reconociéndose que las "mayores diferencias surjan probablemente a la hora de definir estrategias y programas dirigidos al logro de esta transformación". Si la Universidad aspira a convertirse en factor de cambios (en función que hace a su esencia, en tanto tiene como misión hacer una lectura crítica de la sociedad que le permita identificar los problemas, formular inteligentemente las preguntas e intensificar la búsqueda de respuestas), debe también ser rigurosamente crítica de su propio funcionamiento, más teniendo en cuenta la necesidad actual de aspirar a un mayor grado de legitimación social, especialmente en sus comportamientos, modos de funcionamiento y concreción de sus funciones y objetivos. Y esa necesidad debe potenciar el sentimiento de pertenencia y convertirse en disparador de un proceso de reflexión crítica y de cambios que nos lleven a preservar la tradición que la Universidad nacional y pública ha tenido y tiene en la vida y desarrollo de nuestro país".

\section{Del sistema de Concursos a la Carrera Docente en la UNR (1)}

Un aspecto fundamental de la evaluación de la calidad de las universidades, es la evaluación de la función docencia. En este sentido, la tradición universitaria se ha apoyado en la evaluación de los docentes a través del sistema de concursos abiertos, públicos por oposición y antecedentes.

El régimen de ingreso, evaluación y permanencia de los docentes en la Universidad Nacional de Rosario se había regido, desde 1984, por Resoluciones y Ordenanzas que establecían que el acceso al cargo docente ordinario era por concurso abierto de oposición y antecedentes, evaluado por un jurado integrado por docentes de reconocida trayectoria en el ámbito universitario e integrado -además- por un miembro graduado y un miembro estudiante. Una vez adjudicado el cargo, las subsiguientes evaluaciones se realizarían por un nuevo concurso a los cuatro años del primero y, sucesivamente, al cumplirse períodos de cuatro años se reabriría el concurso. 
En cuanto al concepto de carrera docente, en un primer momento, la polisemia dio lugar a confusiones. Así, por ejemplo, no pocas veces las discusiones se dificultaban porque algunos entendían por carrera docente los cursos de formación pedagógica. Por ello, una vez iniciada la polémica debieron despejarse las diversas interpretaciones del término. Se acotó la expresión carrera docente para denominar el sistema de ingreso, evaluación y promoción del docente en ejercicio de sus funciones en el ámbito de la educación superior universitaria. Esto excluye, entonces, la utilización de tal denominación para los cursos sistemáticos o asistemáticos, de educación formal o no formal, de grado o postgrado de formación pedagógica de los docentes.

Durante más de un año, el tema fue considerado en los Consejos Directivos, en el Consejo Superior, en la Comisión Especial de la Asamblea Universitaria de Política Universitaria sobre Carrera Docente, en la Comisión General de Política Universitaria, en el Rectorado y los Decanatos y en diversas reuniones de los Cuerpos Universitarios. El 22 de noviembre de 1988 sesionó la Asamblea Universitaria (A.U) para considerar los despachos de Política Universitaria elaborados por la Comisión General de Política Universitaria y por la Comisión Especial de Política Universitaria sobre carrera Docente. El debate sobre el tema de Carrera Docente en aquella sesión de la A.U. fue uno de los más ricos y exigentes en argumentos de los que se han dado en ese ámbito. Hubo diversas posiciones sobre cómo estructurar el régimen de ingreso, evaluación y contralor de la gestión docente. Sin embargo, esencialmente, las posiciones expuestas fueron dos, antagónicas entre sí y -según como fueron desarrolladas y fundamentadas - se presentaron como deliberadamente irreconciliables.

Una de las posiciones -que concitó la mayor adhesión de los docentes y de un sector del cuerpo de graduados y de una fracción minoritaria de los no docentes- puede resumirse como sigue:

1. Concurso abierto de antecedentes y oposición para el ingreso a la función docente.

2. Estabilidad en el cargo en forma vitalicia a partir del ingreso por concurso.

3. Promoción de una categoría a otra superior por concurso de antecedentes y oposición.

La otra posición -que recibió la adhesión mayoritaria del cuerpo estudiantil, del cuerpo no docente, de un sector del cuerpo de graduados y de una fracción minoritaria de docentes- se expresa de la siguiente manera:

1. Concurso abierto de antecedentes y oposición para el ingreso y la promoción en la función docente.

2. Periodicidad de la cátedra; esto es, designación por período limitado (4 ó 7 años) al cabo del cual debe realizarse nuevo concurso abierto de antecedentes y oposición. 
Los defensores de la estabilidad sine die del docente equiparaban el status del docente al de un trabajador no docente, como empleado público tanto como con el ejercicio de la magistratura. Por un lado, se caracteriza la función docente como una actividad laboral que debe gozar de iguales respaldos que el derecho garantiza a todo trabajador; esto es, el docente es un trabajador de la educación y, por lo tanto, se debe resguardar su derecho a la estabilidad por lo que su remoción sólo puede producirse merced a causa justificada y mediante un proceso previo (que, para el caso sería el juicio académico). En este sentido se equipararían las condiciones laborales del docente a la del trabajador no docente, para tomar el caso más próximo. En otro orden, se reconoce que la tarea docente reviste un alto grado de compromiso social, en tanto formador de ciudadanos que deben cumplir funciones de gran responsabilidad como investigadores, profesionales, técnicos y docentes, así como participar directamente en actividades de extensión e investigación científica. En este sentido, se reclama la estabilidad como reaseguro de ese compromiso y liberar al docente de la preocupación por la incertidumbre de su futuro en la función a la que dedica sus esfuerzos. Además, se llegó a cuestionar el sistema del concurso de antecedentes y oposición por considerarlo arbitrario. Otro de los argumentos fuertes es el hecho de considerar que los concursos periódicos, sin consideración por la labor en la cátedra, dificulta la conformación de equipos de cátedra ya que cada renovación pondría en peligro la integración lograda, si se renovaran sus miembros.

Desde la posición contraria, la que sostiene la periodicidad de la cátedra, se argumenta sobre la base de considerar prioritaria la potestad de la universidad para garantizar el sostenimiento y la elevación de la calidad de la enseñanza a través de la evaluación de los docentes.

Desde este principio, se busca la evaluación del docente para asegurar la calidad y la flexibilidad del sistema. En ese sentido, el concurso, por su metodología, garantiza ese objetivo a la vez que asegura el respeto de los derechos del docente. Se afirma que un docente que se ha desempeñado correctamente durante el lapso de su designación y que conoce su materia no puede temer un nuevo concurso. Desde esta posición se afirma que la responsabilidad social que implica la formación docente exige la evaluación periódica.

El sistema que se buscaba pretendía cumplir con las condiciones siguientes:

1. Asegurar el sostenimiento y elevación de la calidad académica.

2. Facilitar la flexibilidad del sistema evitando la consolidación de camarillas o grupos de poder.

3. Garantizar la transparencia y ecuanimidad de procedimientos.

4. Preservar y estimular el esfuerzo y la dedicación de los docentes.

5. Reducir al mínimo posible el margen de incertidumbre en el acceso y permanencia en la función docente. 
6. Contribuir a consolidar los equipos de cátedra.

7. Posibilitar un equilibrio entre la estabilidad y la movilidad del sistema de ingreso y promoción de los docentes.

8. Promover la actualización continua y permanente de los docentes.

9. Contemplar la participación directa de los actores centrales de la relación pedagógica en la evaluación del docente; esto es, docentes y estudiantes.

10. Asegurar un equilibrio entre libertad de cátedra y cumplimiento de los objetivos institucionales.

11. Integrar las tres funciones básicas de la docencia universitaria: docencia, investigación y extensión.

12. Resguardar la dimensión académica de las contingencias de orden político sin desvincular la docencia de la realidad social y política del país, de la educación y de la universidad.

Hasta 1989, en la UNR regía el sistema de concursos periódicos; sin embargo, los concursos no se sustanciaban, los cargos interinos eran (y siguen siendo) un muy alto porcentaje de la planta docente, se asociaba la incorporación al claustro docente con aspectos del "álgebra electoral" y -consecuentemente- la evaluación de la función docencia era una entelequia. En el año 1989, la Asamblea Universitaria aprobó el llamado sistema de la Carrera Docente; sistema que -a través de largos y tortuosos debates- llegó a reglamentar el Consejo Superior. En tal sentido, se aplican distintas normas establecidas en la Ordenanza de Carrera Docente, si bien sólo rigen los aspectos formales del sistema de evaluación y tampoco opera un verdadero sistema de evaluación docente. Mucho menos de evaluación de la gestión institucional de la función docencia. Sin embargo, en los primeros meses de 1997, el Consejo Superior de la UNR. aprobó un Programa de Evaluación Institucional, lo que implicó un ajuste de los sistemas de evaluación de los docentes.

\section{Una breve historia de la carrera docente en la Universidad Nacional de Rosario.}

La UNR cuenta, como expresáramos, con un sistema de evaluación permanente para la docencia que efectiviza la Carrera Docente; a partir del año 1989 la Honorable Asamblea Universitaria definió, en el artículo 60 de su Estatuto, la permanencia de los docentes en sus cargos concursados y dejó sin efecto el concurso periódico, estableciendo el marco normativo que la regula. A partir de entonces el Consejo Superior produjo una serie de normas que regulan su funcionamiento, a saber:

- Ordenanza 526/92 que aprueba el Reglamento de Carrera Docente y De la Formación y Actualización del Docente.

- Ordenanza 527/92 que aprueba el Reglamento De las Funciones y Obligaciones de los Docentes. 
- Ordenanza 528/92 que deja sin efecto el artículo $1^{\circ}$ de las Ordenanzas 526 y 527 y aprueba el Texto Ordenado del Reglamento de Carrera Docente.

- Ordenanza 581/00 que ordena aprobar las disposiciones para la realización de la evaluación del año 2000.

- Ordenanza 583/00 que aprueba el Texto Ordenado de las Disposiciones para la evaluación de la carrera docente a implementarse en el año 2000.

- Ordenanza 588/00 que reforma la Ordenanza 583 en su Artículo $23^{\circ}$ del Anexo II, referido a plazos de recusación.

- Ordenanza 590/01 que modifica la Ordenanza 583 en sus artículos $18^{\circ}$ y $20^{\circ}$ del Anexo II, referido a la composición de las nóminas y comisiones de evaluadores.

- Ordenanza 594/01 que aprueba el modelo de certificado que se extenderá a los docentes evaluados satisfactoriamente.

- Ordenanza 598/01 que modifica la Ordenanza 583 en su Artículo $25^{\circ}$ Anexo II en lo relativo a la ampliación de dictámenes.

Cabe aclarar que es a partir del año 2000 que comienza a ejecutarse con regularidad el proceso de carrera docente. A tal fin se creó una Unidad Ejecutora en el ámbito de la Secretaría Académica de la UNR que coordina con las unidades académicas este proceso y desde entonces se inicia un proceso de análisis de los docentes que trabajan en el ámbito de la UNR: titulares, adjuntos, asociados, jefes de trabajos prácticos y ayudantes. En ese momento, unos 2000 docentes pasaron por esta evaluación a cargo de comisiones externas. El número fue significativo dado que esta evaluación comprendió a aquellos que trabajaban en el ámbito universitario desde la vuelta al período democrático hasta esa fecha. La evaluación que se aplica en el ámbito de la UNR es por juicio de pares, es decir por comisiones interdisciplinarias conformadas por profesores de distintas facultades de la UNR, y también de otras universidades nacionales. En la actualidad se producen convocatorias todos los años para distintos grupos de docentes y la continuidad del sistema está asegurada; la Carrera Docente se rige por la Ordenanza № 602, del 20 de agosto de 2002, producto de sucesivas modificaciones operadas sobre la ordenanza 526/92. La citada ordenanza también tuvo posteriores modificaciones.

\section{Hacia una evaluación del programa de Carrera docente en la UNR.}

Las investigaciones sobre la Universidad como objeto de estudio son relativamente recientes en la atención de los investigadores sociales, al mismo tiempo que los procesos de evaluación institucional en las universidades nacionales han comenzado a desarrollarse, en Argentina, a partir de fines de los ochenta. Desde entonces, se han multiplicado los estudios de casos, la implementación de procesos de evaluación, el debate de las políticas institucionales y la reflexión 
teórica. La Ley de Educación Superior prescribe un ordenamiento básico para establecer un sistema de evaluación de las universidades nacionales - que incluye la evaluación de la función docencia - y plantea algunos mecanismos básicos a partir de la constitución de la Comisión Nacional de Evaluación y Acreditación Universitaria.

La carrera docente de la UNR normalizó su funcionamiento a partir del año 2000 y resulta necesario evaluar su funcionamiento en el ámbito de gestión, a fin de considerar las sugerencias de los actores institucionales implicados. La presente investigación se generó a partir de la detección de núcleos problemáticos en su desarrollo: la organización del sistema de información; el marco normativo; los instrumentos que completa el docente evaluado. A fin de estudiar estos aspectos nos propusimos como objetivo general: diseñar un programa de evaluación para la carrera docente de la UNR; y como objetivos específicos: a) determinar los actores institucionales que intervienen en el proceso y los núcleos problemáticos que estos detectan; b) describir el marco normativo que regula la carrera docente; los formularios e instrumentos que emplea; los procedimientos que aplica y el sistema de información en relación con el aprovechamiento que las unidades académicas y la unidad ejecutora hacen de la información obtenida. El diseño metodológico de la presente investigación involucró a los miembros de la comunidad académica que participan de la carrera docente, en todo el proceso de estudio, a fin de implementar acciones que sean producto de la indagación, de modo de complementar la experticia investigativa con los conocimientos prácticos, vivencias y habilidades de los participantes (Mertens, 2005). Ello permite el análisis los diferentes aspectos de la realidad y la identificación de los actores intervinientes, junto con las relaciones que están detrás de sus experiencias, a través de discusiones y reflexiones sobre informaciones y prácticas específicas, a fin de lograr la generación de nuevos conocimientos para el investigador y los grupos involucrados, abriendo así la posibilidad de aplicar categorías científicas para la comprensión y mejoramiento de la gestión, a partir del trabajo en colaboración de los propios actores involucrados.

Las técnicas empleadas en este proyecto contemplan:

A) Reuniones de reflexión y producción con los actores institucionales identificados. A tal efecto se conformaron diferentes grupos seleccionados de acuerdo al tipo de problemas a abordar. Se consideraron, inicialmente, los siguientes grupos: Grupo sistema de información: responsables de la Unidad Ejecutora, de la función docencia de la AEI, de la Secretaría Académica de la UNR, de la Dirección de Informática (DIU) y de la Dirección de Estadísitica de la UNR; Grupo Marco Normativo: responsables administrativos del Consejo Superior, de Asesoría Jurídica, de la Unidad Ejecutora y de la Comisión Ad-Hoc del Consejo Superior, Comisión de Asuntos Académicos del Consejo Superior; Grupo Actores Institucionales: responsables de carrera docente de las unidades académicas, 
docentes concursados por categoría y dedicación, evaluadores por áreas disciplinares.

B) Encuesta a docentes y evaluadores. Con el propósito de analizar los instrumentos de la carrera docente se aplicó una encuesta para relevar información sobre la suficiencia y pertinencia de los instrumentos empleados. A tal efecto se construyó una muestra aleatoria simple del banco de evaluadores y de los docentes en actividad evaluados.

En lo que se refiere al primer aspecto señalado, la Unidad de Ejecución de Evaluación (UEE) de la mencionada secretaría informó a través de dos de sus miembros - que a su vez pertenecen al equipo de investigación —, que se convocó a la Comisión Ad Hoc de Carrera de Docente del Consejo Superior y a los secretarios académicos de las distintas facultades a fin de resolver problemas referidos al marco normativo. En primer término se consideró la correcta definición de la relación cargo-dedicación. Las funciones y obligaciones del evaluado no están estipuladas según la dedicación sino según el cargo y esto admite un margen de discrecionalidad; se propuso que los evaluadores tengan un criterio unificado para valorar el rendimiento docente en cada eje, según la dedicación. En segundo término la UEE sugirió la incorporación al texto de la ordenanza de aspectos contemplados en la Resolución 294/2000 que regula la situación de los jubilados contratados y los docentes con trámite de jubilación iniciado. También se consideraron otros aspectos, en función de sugerencias realizadas por evaluadores y docentes evaluados: a) que la documentación probatoria del docente sea presentada antes de la reunión de la Comisión Evaluadora; b) que se amplíe el plazo de impugnación del dictamen de la Comisión Evaluadora a diez días hábiles; c) que todos los plazos establecidos en la ordenanza se cuenten como días hábiles; d) que los docentes con licencia - por ejercicio de cargos de gestión o de cargos fuera de la UNR, por enfermedad o por tareas pasivas - no se evalúen; en caso de reincoporarse, que se presenten en la primera convocatoria inmediata posterior, con mediación de dos años para la acumulación de actividades académicas demostrables; e) que los Jefes de Trabajos Prácticos y Ayudantes de Primera sean evaluados por cuatro jurados internos, para explicitar en la ordenanza lo que se hace de manera tradicional. En último término, la UEE propuso la designación de evaluadores por períodos fijos y la constitución de comisiones con miembros rotativos. Las unidades académicas fueron consultadas vía mail —secretarios académicos, integrantes de la Comisión de Asuntos Académicos del Consejo Superior, integrantes de la Comisión Ad Hoc de la Carrera Docente, responsables de Carrera Docente- Las respuestas obtenidas sugieren: a) incorporación al Informe de Evaluación de aspectos contextuales: características curriculares de las asignaturas; carga horaria; modalidad de dictado; cantidad de alumnos; alumnos regulares por cohorte; incorporación de los ejes actividad profesional y formación pedagógica-didáctica; b) incorporación de la opinión de los alumnos 
sobre el desempeño docente; c) entrevista personal entre los miembros de la Comisión Evaluadora y el docente evaluado.

\section{Reflexiones finales}

Muchos autores entienden que la calidad de la formación en la universidad depende en buena medida de la evaluación del personal docente, lo que necesita de una perspectiva evaluativa que vaya más allá de una descripción de lo que el docente realiza y que permita comprender la potencialidad de su tarea, lo cual requiere romper con viejos paradigmas y con el énfasis tecnicista y el carácter positivista que ha acompañado al concepto de evaluación. Saber para qué se evalúa, conocer a quién le interesa y a quién va a servir la evaluación permite la comprensión del fenómeno evaluativo y entender que no se pueden explicar los resultados obtenidos ateniéndose sólo a las características intrínsecas del objeto de evaluación. La evaluación del desempeño docente debe ser asumida como un proceso permanente, enmarcado dentro de una concepción de calidad de la educación y enfocado hacia el perfeccionamiento de la docencia en la Universidad y hacia el mejoramiento de la calidad institucional (Rizo Moreno, 1999).

Los puntos de vista expuestos ponen en evidencia la relevancia de un Programa de Evaluación de Carrera Docente, que explicite las expectativas y logros para la docencia en el ámbito de la UNR, y que contribuya a la formación de los equipos que participen en la evaluación de la carrera docente en los ejes evaluación, planificación y docencia universitaria, al mismo tiempo de permitir la generación de conocimientos sobre evaluación de la profesión docente en la Educación Superior. En este sentido, se espera poder integrar el programa a los procesos de evaluación permanente de la UNR, de modo de participar en la planificación de políticas académicas de formación y consolidación del cuerpo docente. Ello permitirá la conformación de concensos para la determinación de los logros, objetivos y estándares que se persiguen en la docencia universitaria, a partir de la provisión de información a los subsistemas de la UNR para su comunicación a la comunidad académica.

Adecuar la cultura institucional de las universidad a los actuales paradigmas de reforma del sector, exige cambios en los valores y creencias de la comunidad académica que pueden generar conocimientos e interpretaciones compartidos acerca de los acontecimientos de su propia vida interna, de modo de constituir formas generales de percibir, pensar y sentir, a partir de historias internalizadas y conocimientos comunes, componentes del aglutinador común que es la cultura organizacional (Kuh, Whitt, 1998).

La evaluación debe ser emprendida desde una mirada comprensiva e integradora, de modo de impactar en el desempeño docente, aceptándose que la misma no sólo debe cualificarlo, sino también potenciarlo, para poder 
acompañar el desarrollo académico y convertirse en una verdadera fuente de conocimiento para la Universidad; desarrollar investigaciones que permitan implementar programas de evaluación que tengan en cuenta los contextos de trabajo específicos, resulta una necesidad a ser tenida en cuenta en los estudios sobre Educación Superior.

\section{Notas Bibliográficas}

(1) En esta parte del trabajo se toman elementos del Proyecto de Investigación EVALUACIÓN DE LA UNIVERSIDAD: LA FUNCIÓN DOCENCIA. ANÁLISIS DE LOS CRITERIOS EMPLEADOS EN LOS PROCESOS DE EVALUACIÓN DE LOS DOCENTES UNIVERSITARIOS A TRAVÉS DEL SISTEMA DE CONCURSOS Y DE LA CARRERA DOCENTE, Convocatoria Proyectos 1997-1998, Programa de Fomento de la Investigación Científica y Tecnológica (Resol. CS. N ${ }^{\circ}$ 202/92), Secretaría de Ciencia y Tecnología, UNR, Director: Prof. Enrique Barés, Integrantes: Schanzer, R., Martinez de Aguirre, E., Linares, R., Wheeler, C.

\section{Referencias bibliográficas}

- Achilli, E. (2000). Investigación y formación docente. Rosario. Laborde Editor.

- Altbach, P. (2001). Patrones de desarrollo de la educación superior. Educación Superior Comparada. El conocimiento, la universidad y el desarrollo. Buenos Aires. Universidad de Palermo, 32-59.

- Arrechavaleta Guartón, N., Tristá Pérez, B. (1992). Cuba: problemas de la planificación, organización y evaluación de la formación y capacitación del personal docente. La administración y el planeamiento universitario. Universidad de La Habana. Centro de Estudios para el Perfeccionamiento de la Educación Superior. Cuba.

- Fernández Lamarra, N. y Marquina, M. (2008). La profesión académica en argentina: explorando su especificidad en el marco de las tendencias internacionales. Revista Alternativas: serie espacios pedagógicos. Año XIII. № 52. Universidad de San Luis

- Hernandez Madrigal, Pastor (2003). Formación docente en Educación Superior: la experiencia de un modelo de Intervención. Contexto Educativo. Revista Digital de Educación y Nuevas Tecnologías. Año V. № 27

- Kuh,G.D. \& Whitt E.J (1998) The invisible tapestry: culture in american colleges and universities. ASHE-ERIC Higher Education Report № 1 Washington D.C: Association for Study of Higher Education.

- Mertens, D.M. (2005). Research and evaluation in Education and psychology: Integrating diversity with quantitative, qualitative and mixed methods. Thousand Oaks. Sage.

- $\quad$ Rizo Moreno, H. (1999) Evaluación del docente universitario. Revista Electrónica Interuniversitaria de Formación del Profesorado. Obtenido el 2 de marzo de 2010. http:// www.uva.es/aufop/publica/revelfop/99-v2n1.htm.

- Ruiz Lúquez, J. (2000) La investigación y la docencia en la universidad. Universitas 2000. Caracas. Fondo Editorial para el Desarrollo de la Educación Superior.

- Universidad Nacional de Rosario (2002). Autoevaluación institucional. Consideraciones generales. Rosario. UNR Editora. 
- VAIN, P, (1998). Evaluación de la docencia universitaria: un problema complejo.Trabajo elaborado en el marco de la Convocatoria organizada por CONEAU para la realización de trabajos Teório-Metológicos sobre evaluación institucional universitaria

- Zabalza, M. (1990). Evaluación orientada al perfeccionamiento. Revista Española de Pedagogía. № 186. España. 